Palladium-Catalyzed Desulfinative Cross-Couplings

Antoine de Gombert ${ }^{1}$ and Michael C. Willis ${ }^{1}$

${ }^{1}$ Department of Chemistry, Chemistry Research Laboratories, University of Oxford, Oxford OX1 4TA, United Kingdom

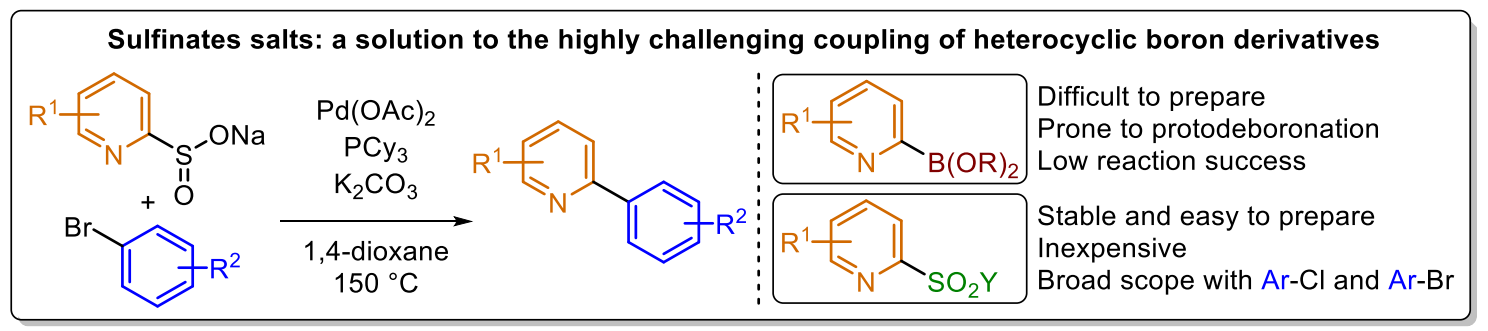

ORIGIN

The Suzuki-Miyaura reaction has undeniably revolutionized the art of synthesis, particularly in the pharmaceutical industry. However, the coupling of 2-pyridine boronic acids, as well as other nitrogenbased heterocycles, has long remained a challenge. In this context, our laboratory has recently reported the use of pyridyl and heteroaryl sulfinate salts as efficient alternatives to boron reagents. This new reaction successfully yields industrially relevant building blocks, the synthesis of which would be challenging using classic boron-based nucleophiles.

\title{
REACTION MECHANISM
}

4-Methylbenzene sulfinate $\mathbf{1}$, pyridine-2-sulfinate $\mathbf{2}$ and 1-bromo-4-fluorobenzene $\mathbf{3}$ were selected as the model substrates for our study $(A)$. The reduction of $\mathrm{Pd}(\mathrm{OAc})_{2}$ in these systems is mediated by the homocoupling of two sulfinate substrates (B). The transmetalation step most likely occurs after dissociation of a phosphine ligand from the oxidative addition complex 4 (C). The counterion of the sulfinate salt has a dramatic effect on the rate of the transmetalation, with $\mathrm{Li}<\mathrm{Na} \ll<\mathrm{K}<\mathrm{Cs}<\mathrm{NMe}_{4}$. We propose that the sulfinate and carbonate salts undergo a cation metathesis prior to the transmetalation, while the carbonate base is also essential for neutralizing the $\mathrm{SO}_{2}$ gaseous by-product. Novel post-transmetalation complexes were isolated and characterized by X-ray crystallography. In order to form a stable square planar arrangement around the palladium center, the complexes formed with carbocyclic sulfinates were found to dimerize (5), or coordinate a second $\mathrm{PCy}_{3}$ ligand (6) (D). In contrast, a 5 -membered metallocycle $\mathbf{7}$ was obtained with pyridine-2-sulfinate $\mathbf{2}$, which can chelate the palladium in a $\mathrm{K}^{2} \mathrm{~N}, \mathrm{o}$-mode. While the transmetalation is the turnover limiting step (TLS) for the reaction involving the carbocyclic sulfinate 1 , it is the extrusion of $\mathrm{SO}_{2}$ that was found to be turnover limiting in the reaction involving pyridine-2-sulfinate $\mathbf{2}(\mathrm{E})$. This kinetic data is in agreement with NMR data, which shows that the resting state intermediate (RSI) of the reaction involving 1 is the oxidative addition complex $\mathbf{4}$, whereas the chelated complex $\mathbf{7}$ was observed to be the resting state intermediate when pyridyl sulfinate $\mathbf{2}$ is employed as the nucleophile. 

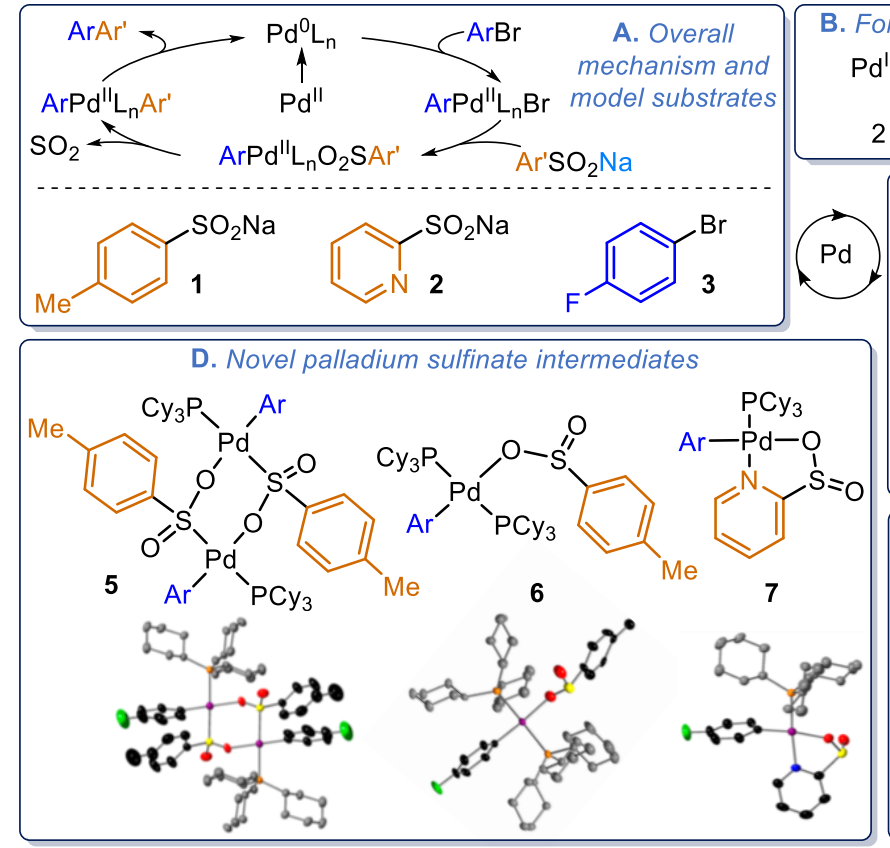

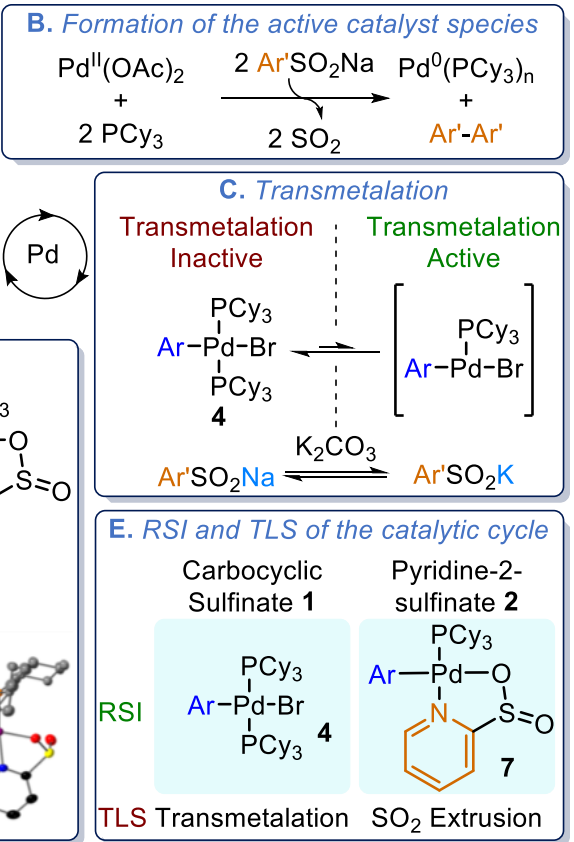

\section{IMPORTANCE}

Despite the robustness and broad scope of this newly developed cross-coupling, the necessity of high temperatures is an issue that needs to be tackled for this method to be more appealing. We hope that this mechanistic insight will encourage interest in these reactions in the scientific community, and lead to the design of more efficient catalytic systems that address the current limitations, and allow these transformations to have a greater impact on the field.

\section{LITERATURE}

$\operatorname{ref}^{1} \operatorname{ref}^{2} \operatorname{ref}^{3} \operatorname{ref}^{4} \operatorname{ref}^{5} \operatorname{ref}^{6} \operatorname{ref}^{7} \operatorname{ref}^{8} \operatorname{ref}^{9} \operatorname{ref}^{10}$

1. de Gombert, A. et al. (2020) Mechanistic Studies of the Palladium-Catalyzed Desulfinative CrossCoupling of Aryl Bromides and (Hetero)Aryl Sulfinate Salts. J. Am. Chem. Soc. 142, 3564-3576

2. Markovic, T. et al. (2017) Pyridine Sulfinates as General Nucleophilic Coupling Partners in Palladium-Catalyzed Cross-Coupling Reactions with Aryl Halides. Chem. Sci. 8, 4437-4442

3. Ortgies, D. H. et al. (2016) Desulfination as an Emerging Strategy in Palladium-Catalyzed C-C Coupling Reactions. Eur. J. Org. Chem. 2016, 408-425

4. Cox, P. A. et al. (2016) Protodeboronation of Heteroaromatic, Vinyl, and Cyclopropyl Boronic Acids: pH-Rate Profiles, Autocatalysis, and Disproportionation. J. Am. Chem. Soc. 138, 9145-9157

5. Blakemore, D. (2016) Chapter 1 Suzuki-Miyaura Coupling. Synthetic Methods in Drug Discovery: Volume 1 1, 1-69

6. Markovic, T. et al. (2017) Catalyst Selection Facilitates the Use of Heterocyclic Sulfinates as General Nucleophilic Coupling Partners in Palladium-Catalyzed Coupling Reactions. Org. Lett. 19, 6033-6035 
7. Markovic, T. et al. (2018) Heterocyclic Allylsulfones as Latent Heteroaryl Nucleophiles in Palladium-Catalyzed Cross-Coupling Reactions. J. Am. Chem. Soc. 140, 15916-15923

8. Sraj, L. O. C. et al. (2012) Who Wins: Pesci, Peters, or Deacon? Intrinsic Reactivity Orders for Organocuprate Formation via Ligand Decomposition. Organometallics 31, 1801-1807

9. Wei, C. S. et al. (2013) The Impact of Palladium(II) Reduction Pathways on the Structure and Activity of Palladium(0) Catalysts. Angew. Chem., Int. Ed. 52, 5822-5826

10. Vitzthum, G. and E. Lindner (1971) Sulfinato Complexes. Angew. Chem. Int. Ed. 10, 315-326 